\title{
Erratum to: The Natural Course of Atopic Dermatitis and the Association with Asthma
}

\author{
Min Qiao, ${ }^{1}$ Deqi Xiao, ${ }^{2}$ Li Qian, ${ }^{3}$ and Jian'ou Qiao ${ }^{3,4,5}$ \\ Erratum to: Inflammation \\ DOI 10.1007/s10753-016-0501-5
}

The original version of this article unfortunately contained a mistake. The author missed to insert the Funding support for their paper. The funding support should read as:

Funding Source. This project was supported by Natural Science Foundation of China General Projects (Project number: 81170028).

The online version of the original article can be found at http://dx.doi.org/ 10.1007/s10753-016-0501-5.

\footnotetext{
${ }^{1}$ Department of Internal Medicine, Kingsbrook Jewish Medical Center, 585 Schenectady Ave, Brooklyn, NY, USA

${ }^{2}$ Department of Dermatology, Shanghai Sixth People's Hospital Affiliated of Shanghai Jiaotong University, Shanghai, China

${ }^{3}$ Department of Emergency, Shanghai Ninth People's Hospital Affiliated of Shanghai Jiaotong University School of Medicine, Shanghai, China

${ }^{4}$ Department of Respiratory Medicine, Shanghai Ninth People's Hospital Affiliated of Shanghai Jiaotong University School of Medicine, No. 639, Zhizaoju RoadHuangpu DistrictShanghai, 200011, China

${ }^{5}$ To whom correspondence should be addressed at Department of Respiratory Medicine, Shanghai Ninth People's Hospital Affiliated of Shanghai Jiaotong University School of Medicine, No. 639, Zhizaoju RoadHuangpu DistrictShanghai, 200011, China. E-mail: qiaojianouhhhhh@ hotmail.com
} 